\title{
Prefrontal Cortical Mechanisms Underlying Individual Differences in Cognitive Flexibility and Stability
}

\author{
Diana J. N. Armbruster ${ }^{1,2}$, Kai Ueltzhöffer ${ }^{1,2}$, Ulrike Basten ${ }^{1}$, \\ and Christian J. Fiebach ${ }^{1,2,3,4}$
}

\begin{abstract}
The pFC is critical for cognitive flexibility (i.e., our ability to flexibly adjust behavior to changing environmental demands), but also for cognitive stability (i.e., our ability to follow behavioral plans in the face of distraction). Behavioral research suggests that individuals differ in their cognitive flexibility and stability, and neurocomputational theories of working memory relate this variability to the concept of attractor stability in recurrently connected neural networks. We introduce a novel task paradigm to simultaneously assess flexible switching between task rules (cognitive flexibility) and task performance in the presence of irrelevant distractors (cognitive stability) and to furthermore assess the individual "spontaneous switching rate" in response to ambiguous stimuli to quantify the individual dispositional cognitive flexibility in a theoretically motivated way (i.e., as a proxy for attractor stabi-
\end{abstract}

\section{INTRODUCTION}

The ability to flexibly adjust our behavior according to a changing environment is crucial for our daily life. However, the successful pursuit of our goals also requires that we can maintain behavior in the face of potential distractors. Thus, cognitive flexibility and cognitive stability are important component processes for the cognitive control of behavior (Banich, 2009; Miyake et al., 2000). A large body of neurocognitive research suggests that a neural network consisting of prefrontal and parietal regions underlies cognitive control (Champod \& Petrides, 2007; Derrfuss, Brass, \& von Cramon, 2004; Curtis \& D'Esposito, 2003; Wager \& Smith, 2003; Collette \& Van der Linden, 2002; Miller \& Cohen, 2001; Collette et al., 1999; D’Esposito et al., 1995). However, whether cognitive flexibility and cognitive stability depend upon separate neural systems or share a common neural network is still a matter of debate (Hedden \& Gabrieli, 2010; Robbins, 2007; Barber \& Carter, 2005; Aron, Monsell, Sahakian, \& Robbins, 2004; Aron, Robbins, \& Poldrack, 2004).

\footnotetext{
${ }^{1}$ Goethe University Frankfurt am Main, ${ }^{2}$ University of Heidelberg, ${ }^{3}$ IDeA Center for Individual Development and Adaptive Education, Frankfurt am Main, Germany, ${ }^{4}$ Radboud University, Nijmegen, The Netherlands
}

lity). Using fMRI in healthy human participants, a common network consisting of parietal and frontal areas was found for task switching and distractor inhibition. More flexible persons showed reduced activation and reduced functional coupling in frontal areas, including the inferior frontal junction, during task switching. Most importantly, the individual spontaneous switching rate antagonistically affected the functional coupling between inferior frontal junction and the superior frontal gyrus during task switching and distractor inhibition, respectively, indicating that individual differences in cognitive flexibility and stability are indeed related to a common prefrontal neural mechanism. We suggest that the concept of attractor stability of prefrontal working memory networks is a meaningful model for individual differences in cognitive stability versus flexibility.
Cognitive flexibility is typically studied using paradigms that require switching between different tasks (Monsell, 2003; Jersild, 1927) - for which the inferior frontal junction area (IFJ), located at the junction of the inferior frontal sulcus (IFS) and the precentral sulcus, has been found to play a key role (Brass, Derrfuss, Forstmann, \& von Cramon, 2005; Derrfuss, Brass, Neumann, \& von Cramon, 2005; Derrfuss et al., 2004). Attention shifting tasks are similar in that they require focusing attention to a different feature or stimulus within the same task rule (Hedden \& Gabrieli, 2010; Wager, Jonides, \& Reading, 2004). Other important operationalizations of cognitive flexibility include rewardor feedback-based learning of rules for which the orbitofrontal cortex as well as the striatum have been indicated to play an important role (e.g., Cools, Clark, Owen, \& Robbins, 2002).

Cognitive stability has also been related to $\mathrm{pFC}$ functioning. Specifically, the dorsolateral pFC (DLPFC) is associated with the formation of distractor-resistant memories (Toepper et al., 2010; Sakai, Rowe, \& Passingham, 2002), and the ventrolateral pFC (VLPFC) is critical for resolving interference from distractors (Jha, Fabian, \& Aguirre, 2004) as well as for the inhibition of responses and task sets (Forstmann et al., 2008; Aron, Robbins, et al., 2004; Konishi et al., 1999). Furthermore, behavioral as well as functional neuroimaging data suggest that individuals also differ in 
their cognitive flexibility and stability (Muller et al., 2007; Hedden \& Yoon, 2006; Wager, Jonides, Smith, \& Nichols, 2005; Miyake et al., 2000). However, as of now, only few neuroimaging studies realized paradigms allowing for the simultaneous assessment of cognitive flexibility and stability (Hedden \& Gabrieli, 2010). To investigate the shared versus segregated cortical representation of cognitive flexibility and stability, it is necessary to study both functions within one task context with the same stimuli.

Complementing the functional neuroimaging data, computational neuroscience has developed models of prefrontal network dynamics that may help to understand the mechanisms underlying cognitive flexibility and cognitive stability (Compte, Brunel, Goldman-Rakic, \& Wang, 2000; Durstewitz, Seamans, \& Sejnowski, 2000; Wang, 1999). Specifically, the concept of recurrently connected neural networks that exhibit multiple stable states is often used to model how the stable maintenance of information over time, in pFC, is achieved (Loh, Rolls, \& Deco, 2007; Durstewitz \& Seamans, 2002; Brunel \& Wang, 2001; Durstewitz et al., 2000; Durstewitz, Kelc, \& Gunturkun, 1999). In such models, the attracting, that is, stable states that correspond to working memory representations or representations of task sets, can be thought of as basins in a potential landscape (Figure 1). Task switching, accordingly, is conceptualized as a transition from one attractor state to another. The depth of the basin, that is, the stability of the attracting state, should be related to cognitive stability and cognitive flexibility in an antagonistic way: Whereas deeper basins of attraction would be equivalent to more stable attracting states that are less likely to be disturbed by distracting stimuli or pure neuronal noise, a conscious switch from one basin to another needs less effort for shallower basins.

In the dual state theory of Durstewitz and Seamans (2008), system states that facilitate cognitive stability versus cognitive flexibility are characterized by differential activity

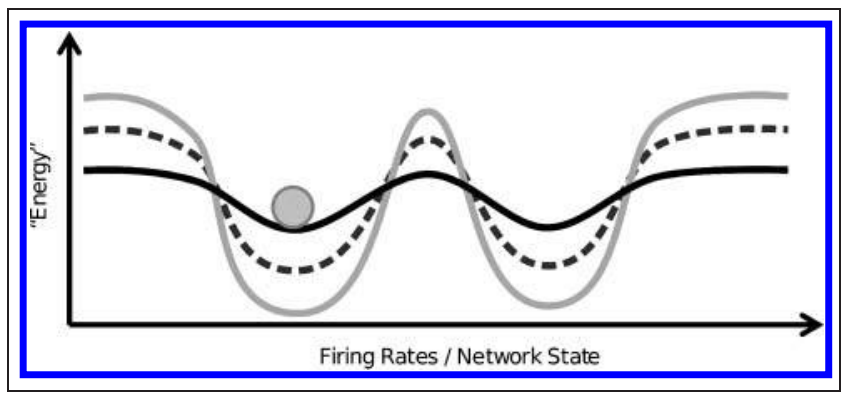

Figure 1. Potential landscape. Stable attractors can be conceptualized as basins in a potential landscape. The basin depth indicates the stability of an attractor, that is, the amount of external forcing that is needed to change the state of the system. Here we schematically show the one-dimensional potential landscape of a quite flexible system (black) where only a small potential barrier separates two neighboring basins of attraction, a very stable but less flexible system (light gray), and an intermediate system (dotted line). Figure adapted from Durstewitz and Seamans (2008) of dopaminergic subsystems and rely on multiple variables, such as receptor densities (Durstewitz \& Seamans, 2002), baseline neurotransmitter levels (Durstewitz \& Seamans, 2008; Thurley, Senn, \& Luscher, 2008), or the efficiency of neurotransmitter clearance (Bilder, Volavka, Lachman, \& Grace, 2004). Thus, it can be derived from computational models that the attractor properties should vary between persons. For example, genetic variation of neurotransmitter systems between persons may influence the depth of attractor states (Durstewitz \& Seamans, 2008; Bilder et al., 2004), and these network properties propagate to the behavioral correlates of cognitive stability and flexibility (van Holstein et al., 2011; Rosa, Dickinson, Apud, Weinberger, \& Elvevag, 2010; Stelzel, Basten, Montag, Reuter, \& Fiebach, 2009, 2010). Different attractor properties have also been suggested to play a role in psychopathology. For example, extremely shallow or extremely deep attractor states of prefrontal networks might be related to the origin of positive and negative symptoms in schizophrenia, respectively (Durstewitz \& Seamans, 2008; Rolls, Loh, Deco, \& Winterer, 2008; Loh et al., 2007). Thus, to summarize, from a neurocomputational perspective, we can derive the hypothesis that cognitive stability and cognitive flexibility are antagonistically related and that persons differ in their degree of cognitive flexibility.

We reasoned that individual differences in attractor stability-i.e., in the individual depth of the attractor states of prefrontal task networks — may be adequately measured by how readily individuals switch from one task state to another in the light of ambiguous task cues. This measure reflects the probability of spontaneously switching from one attracting state to another without an explicit external cue, thus we refer to it as the "individual spontaneous switching rate." According to this antagonistic or dimensional model of cognitive flexibility and cognitive stability, we expected to find opposing trends in behavior and brain activations for individuals with relatively higher versus lower spontaneous switching rates: persons with a greater tendency toward switching (i.e., more flexible persons) should switch tasks faster and more accurately but would also be more prone to distraction. In contrast, persons switching rather rarely under ambiguous conditions (i.e., more stable persons) might be better able to resist distraction, but they might also switch less efficiently and less accurately. Importantly, at the neural level, support for this dimensional model of cognitive stability versus flexibility requires that we identify brain systems that show opposite effects of spontaneous switching rates depending on the cognitive demand, that is, task switching versus distractor inhibition. Thus, we studied how individual differences in the spontaneous switching rate influence behavior and brain activation patterns during task switching and distractor inhibition. As individual differences may influence regional brain activation strengths and interregional functional coupling, we examined both parameters. 


\section{METHODS}

\section{Participants}

Twenty-six participants took part in the study. All had normal vision, reported no history of any neurological or psychiatric diseases, were right-handed, and gave written informed consent according to a protocol approved by the ethics committee of the University of Heidelberg. One participant was excluded due to technical problems with response recording. A further five participants were excluded from fMRI data analyses, as their error rates were higher than $30 \%$ in at least one condition. Thus, data of 20 participants were entered into the final fMRI analyses (10 men; age $=20-32$ years, mean age $=23.5$ years $)$.

\section{Behavioral Procedure}

A task was developed in which participants had to respond fast and accurately by button presses to digits between 1 and 9 (excluding 5) that were presented in different shades of gray against a black background (Figure 2). Trials had a fixed duration of $2 \mathrm{sec}$ (see below for more details), with stimuli presented for 900 msec. Responses were registered during the whole trial period. In $80 \%$ of the trials, only one digit was shown above the fixation cross using a constant, medium gray value (127, ongoing task). Participants had to decide whether this digit was odd or even and responded with the index/middle finger of the right hand.

For the remaining 20\% of trials, two digits appeared on the screen, that is, one above and one below the fixation cross. In this case, the gray value (i.e., the relative brightness) of the digits indicated which rule applied: In the distractor inhibition condition, the upper digit was brighter (gray value randomly sampled from the interval $[169 ; 195]$ ) than the lower digit (gray value $=[255-$ gray value of the upper digit]). In this condition, participants were instructed to continue using the odd/even decision rule applied to the upper digit. However, in the task switch condition, the lower digit was brighter (gray value randomly sampled from the interval $[169 ; 195])$ than the upper digit (gray value $=[255-$ gray value of the lower digit]), which signaled participants to switch from the upper to the lower digit and to decide whether it was smaller or larger than 5. Participants were instructed and trained such as to always use the brighter of the two digits for task performance. In switch trials, the response had to be given with the index/middle finger of the left hand. After every critical trial (i.e., task switch or distractor inhibition), a series of at least three ongoing task trials followed before the next task trial appeared. Assignment

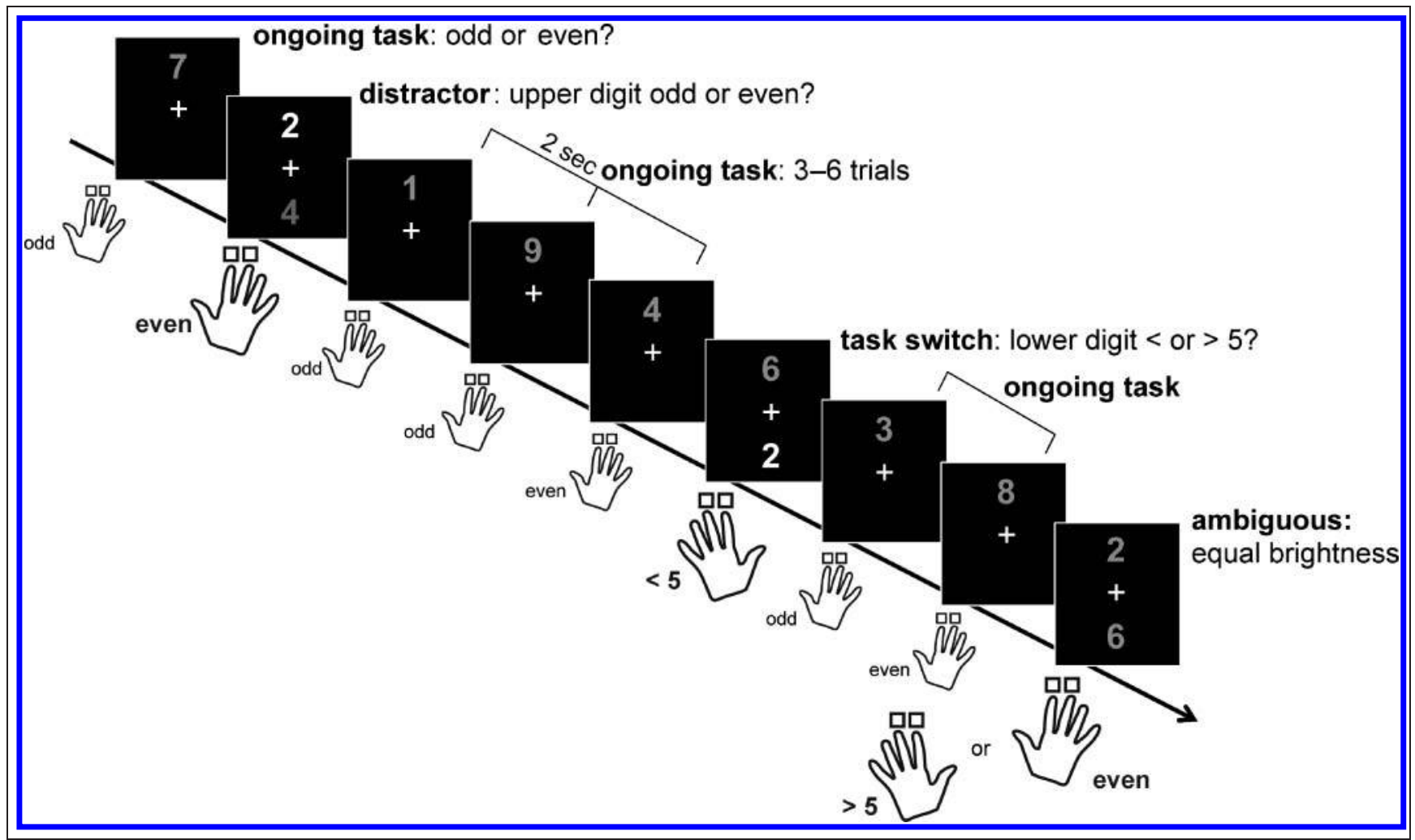

Figure 2. Schematic illustration of the task. Whether responses to the ongoing task were given with the right or left hand (and task switch responses with the other hand, respectively) was counterbalanced across subjects. Here, the former case is shown exemplarily. Most of the trials required a response to only one (upper) digit deciding whether it is odd or even. In $20 \%$ of the trials, a second (bottom) digit appeared. Then, subjects had to ignore this bottom digit if it were darker than the upper digit and continue on the upper digit. If the bottom digit, however, was brighter than the upper digit, subjects had to switch to the bottom digit and respond to it according to the $\langle/>5$ rule with the respective other hand. 
of the task rules to the hands was counterbalanced across subjects.

Finally, in an ambiguous condition, the grayscale values of the two digits were almost identical, such that it was impossible to decide by vision which digit was brighter. Grayscale values of the lower digit were selected randomly from a predefined range (117-137) around the middle grayscale value of the upper digit (which was identical to the one used in the ongoing task, i.e., 127). The slight variation of the grayscale values of the ambiguous lower stimuli along a continuum of $8.2 \%$ of the brightness range aimed at avoiding that participants consciously categorized this condition as ambiguous-as this could have resulted in strategic response behavior. The reasoning behind the ambiguous condition was to assess the individual participant's number of task switches in this condition, which we here call spontaneous switching rate and which reflects the individual shallowness of the attractor state representing the ongoing task rule, and as such constitutes a novel measure of the dimension cognitive flexibility-stability (see Introduction for theoretical background).

Digits and conditions were presented in a pseudorandomized manner with a minimum of three and a maximum of six ongoing task trials presented between two critical trials (i.e., task switch, distractor inhibition, or ambiguous trials). This allowed for modeling the slow hemodynamic response elicited by the critical task trials, whereas the frequent ongoing trials served as implicit baseline (Pollmann, Dove, Yves von Cramon, \& Wiggins, 2000). Before entering the MRI scanner, subjects underwent an instruction and training session lasting approximately $25 \mathrm{~min}$.

\section{fMRI Procedure}

All images were acquired on a 3-T Siemens Trio MRI scanner equipped with a fast gradient system for EPI and a 32-channel head coil. Participants were instructed to lie as quiescently as possible, and their heads were additionally stabilized with cushions. Three hundred ten functional volumes were acquired in a single run lasting approximately 10.5 min, using a T2*-weighted BOLD-sensitive gradientecho, EPI sequence with 32 oblique axial slices (thickness = $3 \mathrm{~mm}$, interslice gap $=1 \mathrm{~mm}$, field of view $=192 \mathrm{~mm}$, matrix size $=64 \times 64$, in-plane resolution $=3 \times 3 \mathrm{~mm}$, repetition time $=2000 \mathrm{msec}$, echo time $=30 \mathrm{msec}$, flip angle $=80^{\circ}$ ). The first three volumes were discarded to allow for stable magnetization. In addition, a T1-weighted magnetization prepared-rapid gradient echo scan was acquired (thickness $=1 \mathrm{~mm}$, field of view $=256 \mathrm{~mm}$, matrix size $=256 \times 256$, in-plane resolution $=1 \times 1 \mathrm{~mm}$, repetition time $=1570 \mathrm{msec}$, echo time $=2.63 \mathrm{msec}$, flip angle $=30^{\circ}$ ).

\section{fMRI Data Analysis}

fMRI data were analyzed using the Statistical Parametric Mapping software (SPM8; www.fil.ion.ucl.ac.uk/spm/software/ spm8/). EPI images were first slice-time and motion corrected. Segmentation of the structural image provided normalization parameters that were used to normalize the functional images to the Montreal National Institute (MNI) template reference brain. Finally, images were smoothed with an 8-mm FWHM Gaussian kernel.

For statistical analyses, a general linear model using a canonical hemodynamic response function and a high-pass filter with $128 \mathrm{sec}$ cutoff (Friston et al., 1998) was applied including one regressor for the task switch and one for the distractor inhibition condition. The ambiguous condition was split into two regressors, one modeling those trials in which participants had switched and one in which they had chosen to stay with the ongoing task. In addition, one regressor was entered for error trials, and six regressors modeled the motion parameters derived from the preprocessing. The ongoing task trials served as implicit baseline (Pollmann et al., 2000) and were not modeled explicitly. For the analysis of individual differences, the individual spontaneous switching rate-i.e., the number of task switches in the ambiguous condition-was entered as covariate into the group-level random-effects model to identify brain regions whose activity covaries with the individual spontaneous switching rate.

Throughout the analyses, a significance level of 5\% (family-wise correction for multiple comparisons) was applied. To this end, a voxel-level threshold of $p<.005$ (uncorrected) was combined with a nonarbitrary clusterextent threshold. The reasoning behind this is that voxel values in fMRI data are not independent from each other, which should result in spatially extended clusters of significantly activated voxels (Forman et al., 1995). The clusterextent threshold $(k=141)$ resulting in a corrected probability of $5 \%$ for false positives was determined via a Monte Carlo simulation with 10,000 iterations using the AFNI routine AlphaSim (Ward, 2000) to model the entire functional image matrix.

\section{Functional Connectivity Analysis}

To explore if the psychological processes of task switching and distractor inhibition result in differential functional connectivity patterns between the involved brain regions, psycho-physiological interaction (PPI) analyses were conducted (Friston et al., 1997). The PPI approach aims at finding brain regions that are differentially coupled with the seed region, depending on specific psychological conditions. The IFJ area was chosen as seed for the PPI analyses as (i) it was shown to be critical for the updating of task representations (Derrfuss et al., 2005); (ii) its activation during task switching is modulated by genetically determined D2 receptor density (Stelzel et al., 2010), which directly relates it to the computational model that motivates the present work; (iii) it was activated for both task switching and distractor inhibition in the present study; and (iv) its activation was modulated by the individual spontaneous switching rate (cf. Results). The exact seed coordinates 
were determined by a conjunction analysis (Nichols, Brett, Andersson, Wager, \& Poline, 2005) of three statistical maps, that is, activation during task switching, activation during distractor inhibition, and the modulatory effect of the spontaneous switching rate during task switching (MNI coordinates $x=-42, y=2, z=+36$, cluster size $=61$ ). The modulatory effect of the individual spontaneous switching rate was assessed by including this variable (see above) as an individual differences predictor in the group analysis, analogous to the univariate analyses of activation strength. PPI results were thresholded using the same procedure as for the univariate analyses, that is, resulting in a corrected $p$ value of .05 .

\section{RESULTS}

\section{Behavioral Results}

Descriptive statistics can be found in Table 1. ANOVAs showed that the task condition had a significant effect on RTs, $F(2,38)=55.7, p=.001$, as well as on error rates, $F(2,38)=4.9, p=.01$. Post hoc $t$ tests (using a Bonferronicorrected significance threshold of $p=.05 / 7=.007$ ) revealed significantly higher RTs in the distractor inhibition compared to the ongoing condition, $T(19)=8.9, p<.007$, and in the task switch compared to the ongoing condition, $T(19)=9.9, p<.007$. Furthermore, RTs were significantly prolonged for the task switch condition compared with the distractor inhibition condition, $T(19)=3.1, p<.007$. Ambiguous cue trials resulted in significantly longer RTs compared with every other condition (baseline vs. ambiguous: $T(19)=$ -10.4 ; distractor vs. ambiguous: $T(19)=-6.2$; task switch vs. ambiguous: $T(19)=-5.4$; all $p<.007$ ), whereas there was no significant difference between ambiguous switch trials and ambiguous non-switch trials, $T(19)=1.2, p=.26$.

Error rates were significantly higher for distractor inhibition compared with the ongoing task, $T(19)=3.3, p<$ .017 , whereas task switching only showed a trend toward higher error rates compared with the ongoing task, $T(19)=$ $2.0, p=.06$. However, when including those five subjects who had to be excluded from the fMRI analyses due to high error rates, the difference was significant, $T(24)=$

Table 1. Descriptive Statistics for Behavioral Data

\begin{tabular}{lcccccc}
\hline & \multicolumn{2}{c}{$R T$ RT (msec) } & & \multicolumn{2}{c}{ Error Rates (\%) } \\
\cline { 2 - 3 } & Mean & $S E$ & & Mean & $S E$ \\
\hline Ongoing task & 698.1 & 26.0 & & 3.7 & 1.3 \\
Distractor inhibition & 870.1 & 18.1 & & 9.0 & 0.7 \\
Task switching & 960.0 & 23.1 & & 7.3 & 1.6 \\
Ambiguous switch & 1135.2 & 31.4 & & 15.3 & 2.4 \\
Ambiguous non-switch & 1105.2 & 31.2 & & 20.1 & 3.3 \\
\hline
\end{tabular}

Standard error was computed according to Cousineau (2005), i.e., the between subject variance was removed as a repeated-measure ANOVA was applied for data analysis.

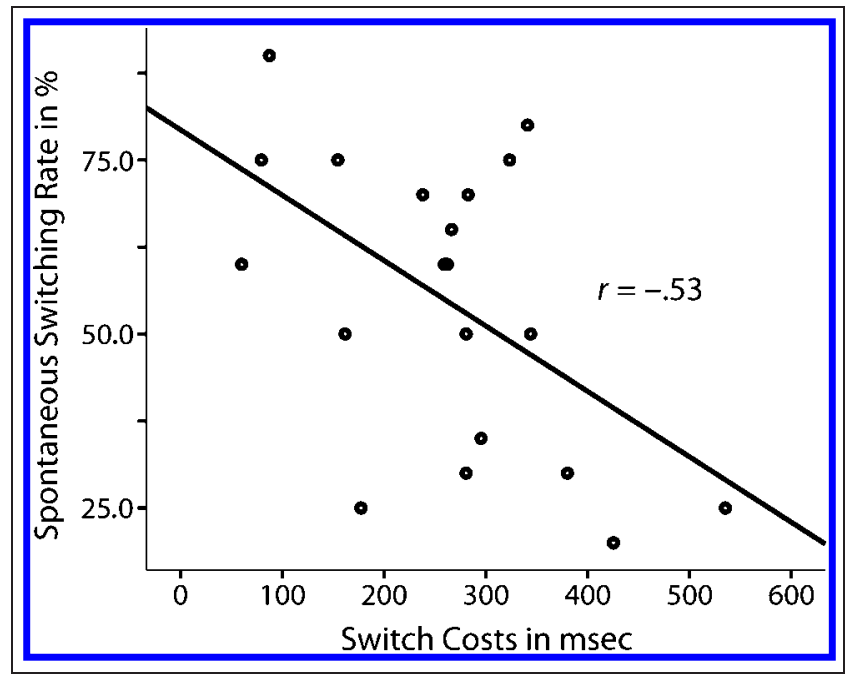

Figure 3. Negative correlation between RT costs for task switching relative to the ongoing task and spontaneous switching rate.

$3.1, p=.005$. There was no difference in error rates between the task switch and the distractor inhibition condition, $T(19)=1.0, p=.35$, with additional subjects $(n=$ $25): T(24)=1.3, p=.21$. The average spontaneous switching rate in the ambiguous condition was 54.8\%, ranging from $20 \%$ to $90 \%$, indicating the presence of sufficient variability in the spontaneous switching rate for individualdifferences analyses.

Correlation analyses showed a significant negative correlation of $r=-.53(p=.02)$ between the spontaneous switching rate and the RT costs of task switching under nonambiguous conditions (Figure 3). For distractor inhibition, the results were less clear: only when the full sample of 25 subjects was considered, the correlation between error rate and the spontaneous switching rate was significant $(r=.45, p=.03$; in the smaller sample $(n=$ 20): $r=.23$; $p=.33)$; the correlation between switch costs and the spontaneous switching rate in this group remained significant $(r=-.53, p=.006)$.

\section{fMRI Results}

\section{Common Network for Task Switching and} Distractor Inbibition

A broad frontoparietal network was active for task switching as well as for distractor inhibition, as compared with the ongoing baseline condition. This network includes lateral and medial frontal areas, parietal and occipital cortex, and subcortical regions (Figure 4A; Table 2). Specifically, both task conditions recruited bilateral posterior frontal cortex including the IFJ, the DLPFC along the IFS, anterior insula with activation extending into the inferior frontal gyrus (IFG) during task switching, superior frontal gyrus (SFG), anterior cingulate cortex (ACC), supplementary motor area (SMA) as well as pre-SMA, and intraparietal sulcus (IPS) extending into the precuneus. Also, occipital 
regions and the cerebellum were significantly activated in both task conditions. Activation of subcortical regions, that is, the thalamus and basal ganglia, was observed only for task switching.

\section{Condition-specific Activation Effects}

Increased activation for task switching relative to distractor inhibition was observed in several of the abovedescribed areas, that is, left IFJ, pre- and postcentral regions extending to the midportion of the insula and the rolandic operculum, dorsal ACC, SMA and pre-SMA, as well as in the occipital lobe. In addition, areas in the superior frontal cortex, ventral ACC, and posterior cingulate cortex were significantly more active for task switching as compared with distractor inhibition (Table 3). Finally, subcortical areas such as the thalamus and especially the basal ganglia, including the dorsal striatum, were activated more strongly for task switching. In contrast, no brain region showed significantly stronger activation for distractor inhibition than for task switching.

\section{Spontaneous Switching Rate Modulates Condition-specific Activations}

A subset of the regions activated for task switching was modulated by the spontaneous switching rate, that is, by the individual number of switches in the ambiguous condition. Specifically, activation in lateral and medial frontal areas including bilateral IFJ, as well as in left-lateralized parietal areas and in the left basal ganglia was reduced in persons with higher spontaneous switching rates (Figure $4 \mathrm{~B}$; Table 4). Thus, persons that are cognitively more flexible in the sense that they are more ready to switch spontaneously under ambiguous conditions activated a subset of the task switching specific regions to a lesser degree during task switching. Notably, the spontaneous switching rate explained variation in task activation beyond what could also be explained by individuals differences in switch costs, as activation in all described brain regions - with the exception of the right IFG-were modulated by the spontaneous switching rate also when the individual switch costs were statistically controlled for (i.e., by inclusion as covariate of no interest in a separate analysis). Brain activity during distractor inhibition (vs. ongoing task), in contrast, was not modulated by individual differences in spontaneous switching rates.

\section{Functional Connectivity Analyses}

To further characterize condition-specific neural networks and their modulation by the individual spontaneous switching rate, we conducted PPI analyses to examine the functional connectivity of task-relevant regions using the left

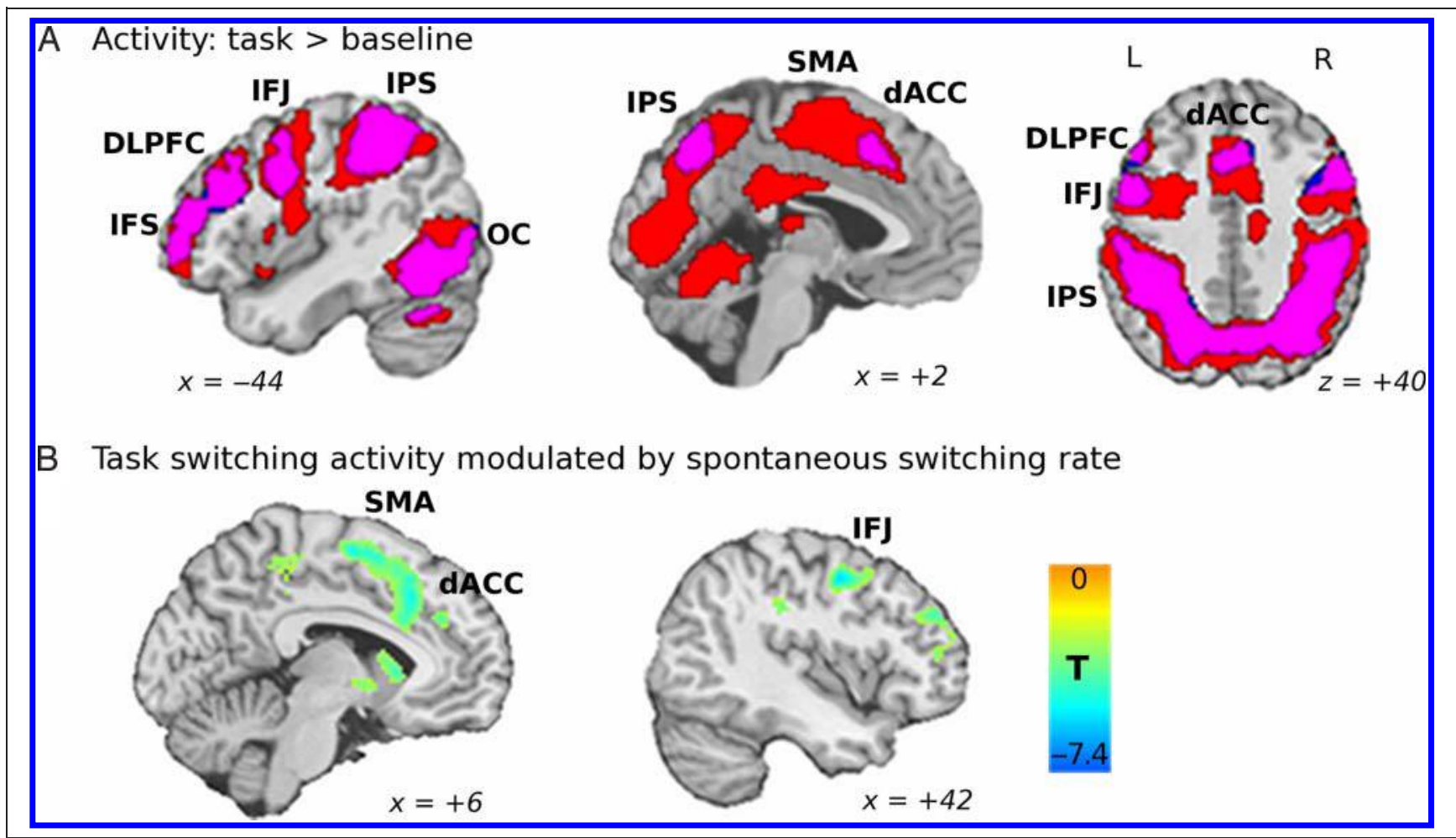

Figure 4. (A) Brain activation for task switching (red) and distractor inhibition (blue); overlap in magenta. (B) Task switching associated brain regions covarying with the spontaneous switching rate. All results displayed at $p<.005, k>141$. dACC $=$ dorsal anterior cingulate cortex, DLPFC $=$ dorsolateral $\mathrm{pFC}, \mathrm{IFJ}=$ inferior frontal junction, IFS $=$ inferior frontal sulcus, IPS $=$ intraparietal sulcus, OC $=$ occipital lobe, SMA $=$ supplementary motor area. 
Table 2. Brain Activation for Task Switching and Distractor Inhibition, as Compared with the Baseline Established by the Ongoing Task Condition

\begin{tabular}{|c|c|c|c|c|c|c|c|}
\hline \multirow[b]{2}{*}{ Brain Region } & \multirow[b]{2}{*}{$B A$} & \multirow[b]{2}{*}{ Hemisphere } & \multicolumn{3}{|c|}{ MNI Coordinates } & \multirow[b]{2}{*}{$T_{\max }$} & \multirow[b]{2}{*}{ Cluster Size } \\
\hline & & & $x$ & $y$ & $z$ & & \\
\hline \multicolumn{8}{|l|}{ Task Switching } \\
\hline \multirow[t]{2}{*}{ Inferior frontal junction (IFJ) } & $9 / 45 / 8 / 6$ & left & -46 & -2 & +44 & 6.39 & 60516 \\
\hline & $9 / 45 / 8 / 6$ & right & +44 & +4 & +34 & 4.77 & a \\
\hline \multirow[t]{3}{*}{ Dorsolateral pFC (DLPFC) } & $9 / 46 / 8 / 6$ & left & -46 & +32 & +36 & 5.63 & a \\
\hline & $9 / 46 / 8 / 6$ & right & +44 & +30 & +30 & 4.59 & a \\
\hline & $9 / 46 / 8 / 6$ & right & +42 & +42 & +12 & 7.21 & a \\
\hline Anterior insula, frontal operculum/inferior frontal gyrus (IFG) & $44 / 45 / 47$ & right & +32 & +22 & -4 & 4.99 & a \\
\hline Supplementary premotor area (SMA)/pre-SMA & $32 / 6 / 8$ & left/right & 0 & 0 & 56 & 5.10 & a \\
\hline Anterior cingulate cortex (ACC) & $24 / 6$ & left/right & -6 & 16 & 42 & 5.11 & a \\
\hline \multirow[t]{2}{*}{ Intraparietal sulcus (IPS)/precuneus } & $40 / 39 / 7$ & left & -30 & -52 & +46 & 7.81 & a \\
\hline & $40 / 39 / 7$ & right & +54 & -36 & +52 & 9.65 & a \\
\hline Occipital lobe & 17 & left/right & -2 & -84 & +8 & 6.74 & a \\
\hline \multirow[t]{2}{*}{ Basal ganglia (globus pallidus, putamen, caudate nucleus) } & & left & -20 & 0 & +14 & 4.65 & a \\
\hline & & right & +18 & +2 & +14 & 4.56 & a \\
\hline Thalamus & & right & +18 & -22 & +4 & 8.13 & a \\
\hline Cerebellum & & left & -2 & -60 & -12 & 7.90 & a \\
\hline \multicolumn{8}{|l|}{ Distractor Inbibition } \\
\hline \multirow[t]{4}{*}{ Inferior frontal junction (IFJ)/dorsolateral pFC (DLPFC) } & $9 / 45 / 8 / 6$ & left & -48 & +4 & +36 & 5.13 & 2853 \\
\hline & & & -44 & +30 & +24 & 4.81 & \\
\hline & $9 / 45 / 8 / 6$ & right & +40 & +4 & +30 & 5.05 & 2909 \\
\hline & & & +46 & +42 & +12 & 4.81 & \\
\hline Anterior insula & & right & +34 & +24 & -6 & 4.08 & 206 \\
\hline $\begin{array}{l}\text { Pre-supplementary premotor area (pre-SMA)/anterior cingulate } \\
\text { cortex (ACC) }\end{array}$ & $32 / 6 / 8 / 9$ & left/right & -6 & +16 & +46 & 4.52 & 445 \\
\hline \multirow[t]{3}{*}{ Intraparietal sulcus (IPS)/precuneus/occipital lobe } & $40 / 39 / 7$ & left & -42 & -36 & +40 & 7.91 & 14969 \\
\hline & & & -44 & -70 & -10 & 5.81 & \\
\hline & & right & +46 & -32 & +44 & 6.30 & \\
\hline
\end{tabular}

${ }^{a}$ For task switching, local maxima are reported as the switching effect forms a single, extensive cluster.

IFJ as seed region (cf. Methods section). PPI analyses showed condition-specific increases in functional coupling within task-related functional networks. Thus, for task switching, the left IFJ showed increased coupling with right IFJ, bilateral DLPFC, bilateral IFG, bilateral SFG, the SMA and pre-SMA, bilateral IPS, and inferior parietal cortex, occipital areas, and right cerebellum (Figure 5A, red). Similarly, the left IFJ increased its coupling to the right IFJ, the pars orbitalis of the right IFG, the pre-SMA, bilateral inferior parietal cortex extending into the temporo-parietal junction (TPJ), and the left cerebellum during distractor inhibition (Figure 5A, blue). The direct comparison of the two task conditions showed a specifically increased coupling of the left IFJ, during distractor inhibition, to the right anterior insula extending into the ventro-medial pars orbitalis of the IFG $\left(x=+36, y=+26, z=-6 ; 156\right.$ voxels; $\left.T_{\max }=3.81\right)$ and to the right TPJ $(x=+48, y=-44, z=+42 ; 321$ voxels; $\left.T_{\max }=4.54\right)$. For task switching, there were no specific coupling effects.

Most importantly, the spontaneous switching rate also influenced the condition-specific IFJ coupling. During task switching contrasted to the ongoing task, the strength of functional coupling between left IFJ and left SFG $(x=-22$, $y=+18, z=+46 ; T_{\max }=3.82$ ) was negatively correlated 
Table 3. Brain Activation Associated with Task Switching $>$ Distractor Inhibition

\begin{tabular}{|c|c|c|c|c|c|c|}
\hline \multirow[b]{2}{*}{ Brain Region } & \multirow[b]{2}{*}{$B A$} & \multirow[b]{2}{*}{ Hemisphere } & \multicolumn{3}{|c|}{ MNI Coordinates } & \multirow[b]{2}{*}{$T_{\max }$} \\
\hline & & & $x$ & $y$ & $z$ & \\
\hline Inferior frontal junction (IFJ) & $9 / 45 / 8 / 6$ & left & -44 & -2 & +44 & 4.26 \\
\hline Superior frontal cortex & $6 / 8$ & right & +26 & -8 & +52 & 6.57 \\
\hline \multirow[t]{2}{*}{ Pre-/postcentral gyrus } & $2 / 3 / 4 / 6$ & left & -58 & -28 & +50 & 4.12 \\
\hline & $2 / 3 / 4 / 6$ & right & +56 & -22 & +44 & 6.37 \\
\hline \multirow[t]{2}{*}{ Mid-insula/rolandic operculum } & 22 & left & -54 & -2 & +4 & 5.74 \\
\hline & 22 & right & +50 & 0 & +4 & 5.75 \\
\hline Dorsal anterior cingulate cortex (dACC) & $24 / 6$ & left/right & 0 & +10 & +28 & 6.11 \\
\hline Ventral anterior cingulate cortex (vACC) & 24 & left/right & +2 & +32 & +12 & 6.88 \\
\hline Posterior cingulum & $23 / 31$ & left & -6 & -38 & +24 & 6.21 \\
\hline Supplementary premotor area (SMA)/pre-SMA & $32 / 6 / 8$ & right & +12 & -8 & +64 & 6.80 \\
\hline Occipital lobe & $17 / 18$ & left/right & +10 & -82 & 0 & 13.08 \\
\hline Basal ganglia (globus pallidus, putamen, caudate nucleus) & & right & +24 & +4 & +14 & 4.78 \\
\hline Thalamus & & right & +12 & -18 & +12 & 6.61 \\
\hline
\end{tabular}

Local maxima are reported. Cluster size is not reported for each peak as the whole network forms a single, extensive cluster $(k=47,991$ voxels).

with the spontaneous switching rate, that is, it was weaker in persons with higher spontaneous switching rates (Figure $5 \mathrm{~B}$, red). In contrast, during distractor inhibition as opposed to the ongoing task, the coupling between left IFJ and left frontopolar cortex, left DLPFC (MFG and SFG), right SFG, and parietal regions (precuneus, TPJ) was positively correlated with the spontaneous switching rate (Figure 5B, blue; Table 5). A conjunction of these two individual difference-PPI results showed that exactly one brain region, in the left SFG (at $x=-22, y=+16$, $z=+48 ; k=83$ ), was antagonistically coupled with left IFJ depending on task condition (Figure 5B and 6A). Thus, the effect of the spontaneous switching rate on IFJ-SFG coupling was negative during task switching but positive during distractor inhibition (Figure 6B). When including the individual switch costs as covariate of no interest in a control analysis, the modulatory effect of the spontaneous switching rate on distractor inhibition related coupling was

Table 4. Task Switching Associated Brain Regions Covarying with the Individual Spontaneous Switching Rate

\begin{tabular}{|c|c|c|c|c|c|c|c|}
\hline \multirow[b]{2}{*}{ Brain Region } & \multirow[b]{2}{*}{$B A$} & \multirow[b]{2}{*}{ Hemisphere } & \multicolumn{3}{|c|}{ MNI Coordinates } & \multirow[b]{2}{*}{$T_{\max }$} & \multirow[b]{2}{*}{ Cluster Size } \\
\hline & & & $x$ & $y$ & $z$ & & \\
\hline \multirow[t]{2}{*}{ Inferior frontal junction (IFJ) } & $9 / 45 / 8 / 6$ & left & -36 & +4 & +40 & 4.32 & a \\
\hline & $9 / 8 / 6$ & right & +44 & -2 & +46 & 6.44 & 361 \\
\hline \multirow[t]{2}{*}{ Middle frontal gyrus (MFG) } & $46 / 10$ & left & -34 & +42 & +28 & 6.51 & a \\
\hline & $9 / 10 / 46$ & right & +38 & +48 & +26 & 5.50 & 1260 \\
\hline Inferior frontal gyrus (IFG) & $47 / 44 / 45$ & right & +48 & +20 & -2 & 4.24 & 274 \\
\hline Dorsal anterior cingulate cortex (dACC) & $32 / 24 / 6$ & left/right & -2 & +24 & +36 & 5.23 & a \\
\hline Supplementary premotor area (SMA) & $32 / 6 / 8$ & left/right & -6 & -4 & +54 & 6.44 & a \\
\hline Postcentral gyrus & $3 / 4$ & left & -20 & -32 & 66 & 7.74 & a \\
\hline Inferior parietal cortex (IPC) & 40 & left & -48 & -28 & +26 & 5.99 & a \\
\hline Basal ganglia (globus pallidus, putamen, caudate nucleus) & & left & -10 & +10 & -4 & 5.49 & a \\
\hline
\end{tabular}

${ }^{\mathrm{a}}$ Local maxima are reported within one extensive cluster $(k=12264)$. 
Figure 5. (A) Brain regions coupled to the left IFJ during task switching (red) and during distractor inhibition (blue), both relative to the ongoing task; overlap in magenta. (B) Brain regions coupled to the left IFJ during task switching (red) and during distractor inhibition (blue) differentially depending on the spontaneous switching rate; overlap in magenta. The arrow shows the area where a conjunction analysis revealed reliable overlap between IFJ coupling under task switching and distractor inhibition (see also Figure 6A). All results displayed at $p<.005$, $k>141$. ACC $=$ anterior cingulate cortex; IFJ = inferior frontal junction; IFG = inferior frontal gyrus; IPS = intraparietal sulcus; $\mathrm{MFC}=$ medial frontal cortex; prec $=$ precuneus; SMA = supplementary motor area; TPJ = temporo-parietal junction.

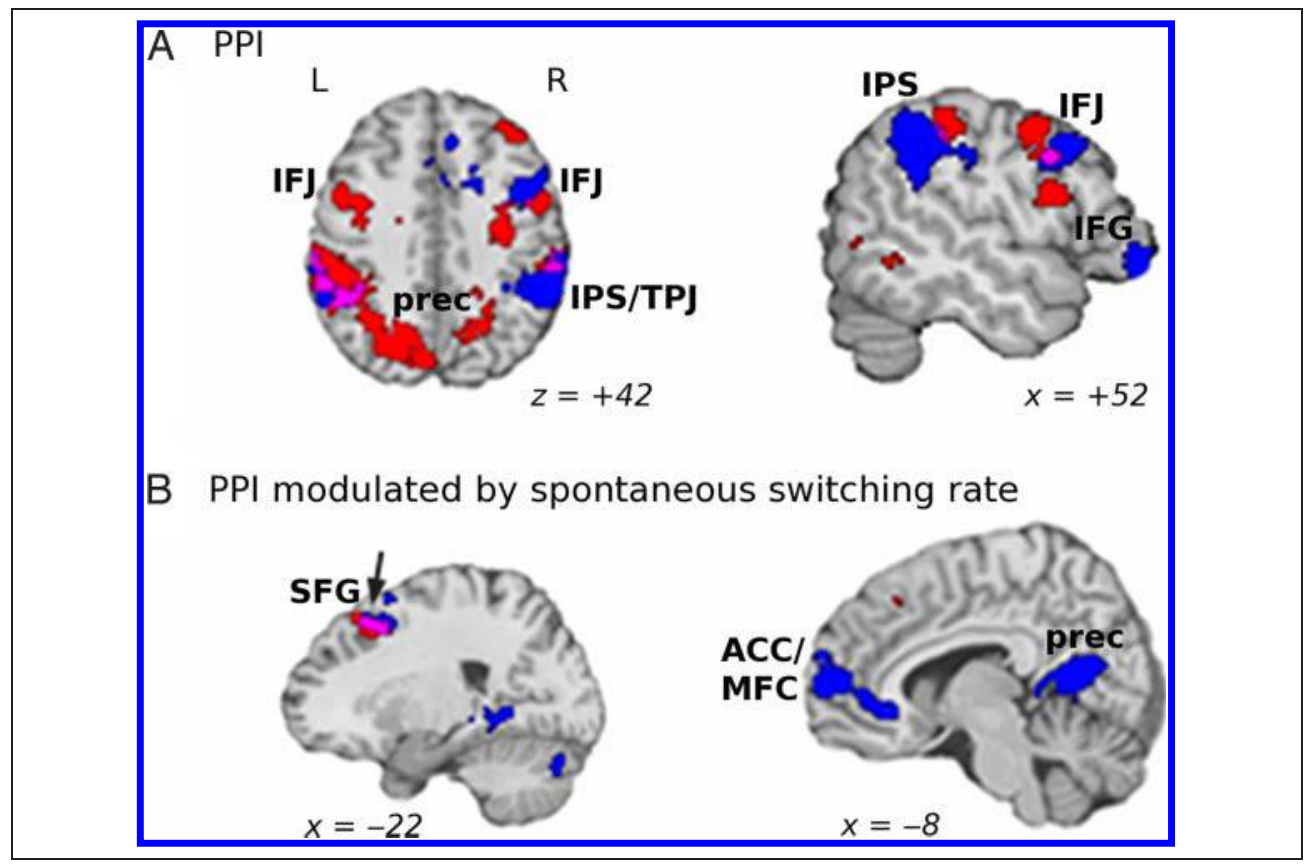

widely unchanged and the effect on task switching related coupling was weaker but still present.

\section{DISCUSSION}

In the present study, we introduce a novel task paradigm that is inspired by neurocomputational modeling of $\mathrm{pFC}$ circuits and that isolates cognitive flexibility (i.e., task switching) and cognitive stability (i.e., distractor inhibition) at comparable levels of difficulty in terms of error rates. Task switching resulted in significantly longer RTs compared with distractor inhibition. This could be due to the fact that task switching requires a greater number of cognitive operations than distractor inhibition, that is, shifting attention to the new stimulus, inhibiting the old task set, updating of the mental representation of the task rule and implementation of a new task set and response (Meiran, Chorev, \& Sapir, 2000), whereas the distractor condition only involves a shift of attention and the inhibition of further task switching-related processes. This is highly consistent with the brain activation data that showed largely overlapping activation in both tasks and increased activity in several areas for task switching compared with distractor inhibition.

Table 5. Brain Regions Coupled to the Right IFJ during Distractor Inhibition Covarying with the Individual Tendency toward Flexibility

\begin{tabular}{|c|c|c|c|c|c|c|c|}
\hline \multirow[b]{2}{*}{ Brain Region } & \multirow[b]{2}{*}{$B A$} & \multirow[b]{2}{*}{ Hemisphere } & \multicolumn{3}{|c|}{ MNI Coordinates } & \multirow[b]{2}{*}{ Voxel $t$} & \multirow[b]{2}{*}{ Cluster Size } \\
\hline & & & $x$ & $y$ & $z$ & & \\
\hline Medial frontal cortex (MFC)/anterior cingulate cortex (ACC) & $10 / 32 / 24$ & left & -10 & +28 & -6 & 4.73 & 943 \\
\hline \multirow[t]{2}{*}{ Superior frontal gyrus (SFG) } & $8 / 6$ & left & -20 & +16 & +50 & 4.12 & 378 \\
\hline & $8 / 6$ & right & +20 & +18 & +48 & 4.22 & 235 \\
\hline Precentral gyrus & $4 / 6 / 9$ & right & +58 & -8 & +36 & 4.63 & 262 \\
\hline \multirow[t]{2}{*}{ Calcarine sulcus/precuneus } & $18 / 19 / 31 / 30$ & left & -10 & -44 & +4 & 5.44 & 1735 \\
\hline & & right & +32 & -58 & 0 & 5.52 & \\
\hline Temporo-parietal junction (TPJ) & $39 / 40$ & right & +40 & -56 & +20 & 3.51 & 227 \\
\hline Cerebellum & & left & -28 & -80 & -30 & 3.90 & 181 \\
\hline
\end{tabular}




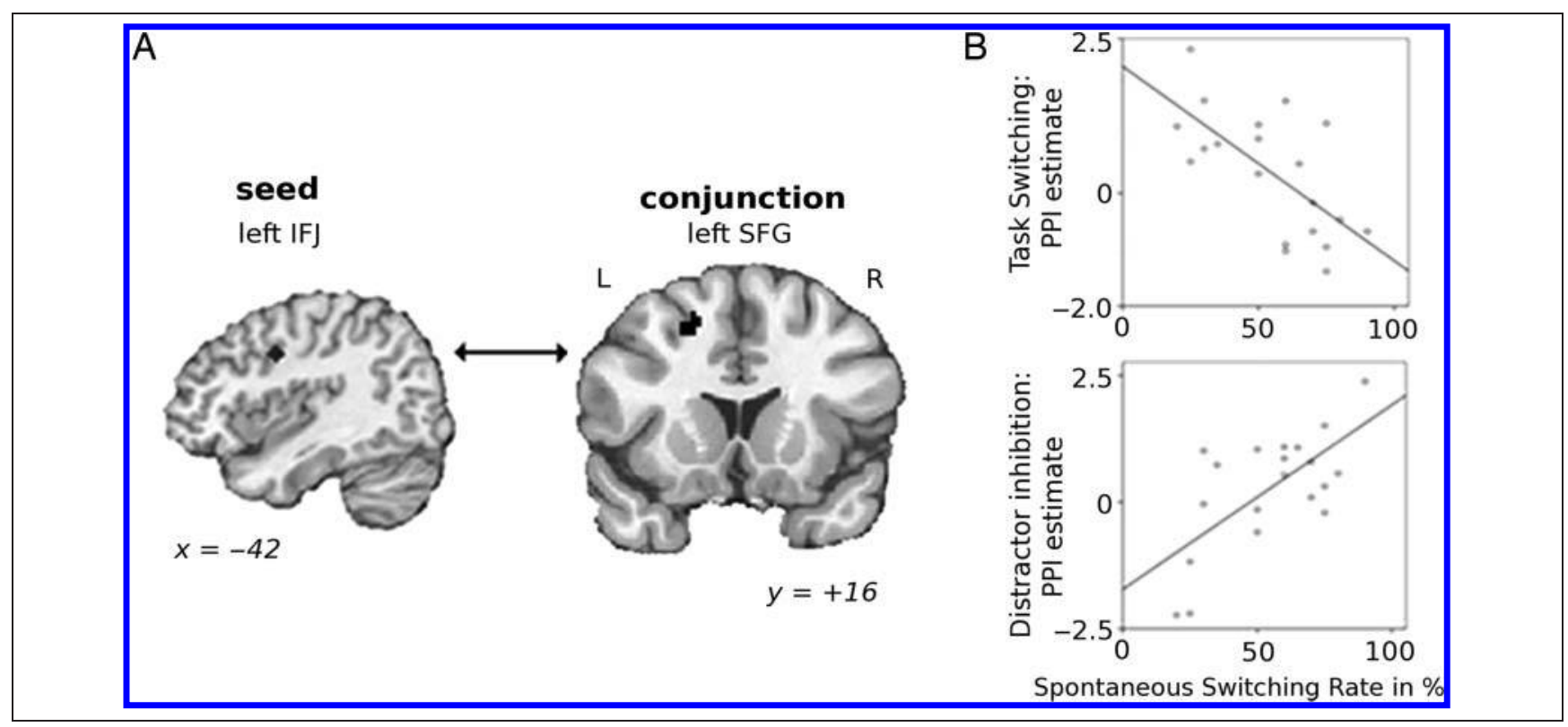

Figure 6. Right SFG $(x=-22, y=+16, z=+48 ; k=83)$ showing an antagonistic modulation of its functional coupling to the left IFJ depending on the spontaneous switching rate and task condition.

In the correlation analyses, some evidence for the dimensional model of cognitive flexibility and stability was found: Results showed that participants exhibiting higher spontaneous switching rates also switched tasks faster than those participants with lower spontaneous switching rates. When the behavioral data of the full sample were considered, there was also a significant correlation supporting the hypothesis that persons switching more often spontaneously are also more distractible, as they tended to produce more errors in distractor trials. These results are consistent with data reported by Dreisbach and colleagues (Dreisbach et al., 2005; Dreisbach \& Goschke, 2004), who also observed higher distractibility in more flexible persons.

\section{Common and Differential Brain Activity and Connectivity for Cognitive Flexibility and Stability}

Consistent with previous studies that separately investigated either cognitive flexibility (Dreher, Koechlin, Ali, \& Grafman, 2002; Dove, Pollmann, Schubert, Wiggins, \& von Cramon, 2000) or cognitive stability (Sakai et al., 2002), we found that a common frontoparietal network is activated by tasks requiring cognitive flexibility and cognitive stability. This network includes lateral prefrontal brain regions, particularly the IFJ, the DLPFC, and regions along the IFS, as well as ACC, SMA, and IPS. Other studies investigating cognitive flexibility and stability simultaneously (Dodds, Morein-Zamir, \& Robbins, 2011; Hedden \& Gabrieli, 2010) also found that most areas of this network were recruited for both cognitive functions, that is, flexibility and stability. The finding of a common neural network for cognitive stability and flexibility is also consistent with the view that a common neural system underlies cognitive control in general (Duncan \& Owen, 2000).
There are two crucial differences between the current study and the only other study that aimed at disentangling the neural correlates of cognitive stability and flexibility (Hedden \& Gabrieli, 2010): the operationalization of cognitive flexibility and the complete lack of IFJ activation in the study of Hedden and Gabrieli (2010). While Hedden and Gabrieli's (2010) paradigm required a shift of attention between global and local features of an object, our paradigm involves multiple levels of switching (task rule, attentional focus, response hand), with the aim of inducing a high demand on cognitive control systems. This might account for the lack of IFJ activation in Hedden and Gabrieli (2010). Although the IFJ was found to be reliably involved in task switching (Derrfuss et al., 2005), even when individual differences in sulcal anatomy were taken into account (Derrfuss, Brass, von Cramon, Lohmann, \& Amunts, 2009), the IFJ seems to be particularly critical for the updating of task rule representations, which is clearly not required when applying the same task rule to global versus local features of the same object.

For the distractor inhibition condition, we did not find any specific activation when compared with task switching. One reason for this might be that, as explained above, less effortful cognitive operations might be necessary for successfully inhibiting a distractor than for successfully switching task rules. In addition, while the sample size of the present study has a typical sample size for human neuroimaging studies $(n=20)$, we also cannot fully exclude a lack of statistical power to detect distractor-specific activations. With respect to the inhibition condition in Hedden and Gabrieli's (2010) study and our distractor inhibition condition, that is, the conditions isolating cognitive stability, both studies found an inferior frontal area and the TPJ to be involved. While Hedden and Gabrieli (2010) 
report stronger VLPFC and TPJ activation, we showedbased on functional connectivity measures - the conditionspecific involvement of TPJ and the anterior insula and IFG/VLPFC during distractor inhibition. These regions are known to be involved in stimulus driven attention (Corbetta \& Shulman, 2002), and VLPFC has further been related to response inhibition processes (Swick, Ashley, \& Turken, 2011; Aron, Robbins, et al., 2004). Given that in our experiment the TPJ and anterior insula/IFG were active in both task conditions, it is plausible to assume that the presentation of the second digit in critical trials triggers a general orienting response toward that stimulus. We speculate that when the stimulus is identified as task-irrelevant, a top-down signal prevents the lower stimulus to gain behavioral relevance, which is mediated by increased coupling of IFJ with anterior insula/IFG and TPJ in the distractor inhibition condition.

To summarize, the analysis of univariate activations and functional connectivities related to cognitive flexibility (task switching) and cognitive stability (distractor inhibition) demonstrates that these two aspects of cognitive control rely to a large degree on shared frontal and parietal brain systems. Depending on the exact nature of cognitive operations involved, brain activation varies with respect to activation strength and/or interareal functional coupling. Against this background, we will now discuss the results for our primary research question, that is, how the recruitment of these systems is modulated by individual differences in the spontaneous switching rate and if our data allow us to draw conclusions concerning the dimensional model of cognitive flexibility and stability.

\section{A Novel Behavioral Measure: Individual Spontaneous Switching Rate}

In this study, we established a novel behavioral measure that is related to the dual state theory of Durstewitz and Seamans (2008) and that aims at capturing the individual stability of attractor states representing the current task rule (cf. Introduction). By placing the subjects in an ambiguous situation, we gave them the possibility to switch task rules while providing only a negligible external cue (due to a minimal variation in the gray values of the stimuli; cf. Methods). The measure of spontaneous switching rate is on the one hand validated by a significant negative correlation with the switch costs in the unambiguous condition (Figure 3). This correlation underlines the fact that spontaneous switching rate is not merely a reflection of a perceptual bias but that it indeed reflects a higher-level cognitive and behavioral tendency toward more flexible behavior. On the other hand, it goes beyond the measure of switch costs by decoupling the individual attractor stability from higher cognitive control processes, which might be confounded in RT costs or error rates in the unambiguous switching condition.

The stability of an attracting state can be defined from a computational perspective as the average time that the system rests within the attractor in the presence of noise (Rolls \& Deco, 2010). Although in purely deterministic theories of dynamical systems (e.g., Strogatz, 2001), attractor stability, and external noise driving the system out of an attracting state can be disambiguated and quantified individually, the intrinsic neuronal noise in the brain, occurring at many levels such as synaptic noise, probabilistic spike times, or network noise (Faisal, Selen, \& Wolpert, 2008), is an integral part of its dynamics and is even believed to be a crucial functional factor for the information processing in the brain (Rolls \& Deco, 2010). It is also the intrinsic noise in the sense of the low activity spontaneous spiking rate that is modulated by GABAergic currents and that, therefore, in the theories of Durstewitz and Seamans (2008) and Rolls et al. (2008), plays a crucial role in controlling the stability of the low-activity attractor state. In this sense, the average resting time in the attracting state in the absence of external forcing is hypothesized to be a good indicator of the attractor stability-also pictured as the depth of the attracting state's basin of attraction in the potential landscape (see also Figure 1) -in the sense of the computational models of cognitive stability, flexibility, and schizophrenia (Durstewitz \& Seamans, 2008; Rolls et al., 2008). This measure is inversely related to the probability of spontaneously switching to another attracting state during a fixed period of time - that is, to the spontaneous switching rate introduced in the present study.

\section{The Individual Spontaneous Switching Rate Modulates Flexibility- and Stability-related Brain Systems}

The functional neuroimaging results showed that individual differences in the spontaneous switching rate (as measured by the number of task switches in the ambiguous condition) modulated brain activity in cognitive control regions like the IFJ. These brain regions were less active during task switching in persons with higher spontaneous switching rates, that is, those persons hypothesized to have rather shallow transitions between different attractor states. While prefrontal neuronal assemblies according to the computational model by Durstewitz and Seamans (2008) are forming attractor states that robustly represent the currently relevant set of task rules, the IFJ-along with other prefrontal, parietal, and striatal brain regions-may be the brain region initiating a different network state. Accordingly, the shallower the energy landscape of the respective neural network, the weaker the signal that IFJ and related regions have to produce in order to effect an updating of the task rule representation. This specific role of the IFJ during rule-based cognitive flexibility is supported by our earlier finding that IFJ activation during task switching, as well as the functional coupling between IFJ and the dorsal striatum, are modulated by the DRD2/ ANKK1-TaqIa polymorphism, that is, by a genetic variation that influences the density of DRD2 receptors in the striatum (Stelzel et al., 2010), which is related to the D1 
receptor activation in pFC (Kellendonk et al., 2006; Laakso et al., 2005). Specifically, we demonstrated that persons with lower D2 receptor densities in the striatum, a state that is associated with increased dopamine activity (Laakso et al., 2005) and decreased D1 receptor activation in PFC, are more efficient in task switching and show less activity in the left IFJ (Stelzel et al., 2010) - the exact region whose activation and functional connectivity patterns were modulated by the spontaneous switching rate in the current study. In line with this, Kellendonk and colleagues (2006) demonstrated in a genetic mouse model of schizophrenia that striatal overexpression of $\mathrm{D} 2$ receptors results in reduced behavioral flexibility. Thus, D2-mediated processes influence cognitive flexibility and its neural correlate in $\mathrm{pFC}$, and the results of the present study suggest that both parameters are related to the robustness of attractor states in prefrontal neural circuits. This conclusion is further supported by the observation that schizophrenic patients exhibit noisier (i.e., less specific) neural activation patterns in tasks such as working memory, oddball, and simple choice RT paradigms (Winterer et al., 2006; Winterer \& Weinberger, 2004). Future work is needed to directly test the association between the spontaneous switching rate and the dopaminergic system in humans.

One important continuation of this line of work will also involve quantifying the relationship between our data and the computational models of cognitive stability and flexibility that motivated the present study. To this end, it will be necessary to develop biophysically plausible, attractorbased models of cognitive tasks like the present one and to directly fit the behavioral and fMRI data by means of physiologically meaningful parameters. This approach will provide a way to quantitatively relate model parameters derived from empirical data to dopamine activity and genetically determined variation in the dopamine system.

\section{Testing the Dimensional Approach of Cognitive Flexibility and Stability}

According to our dimensional model of cognitive flexibility and stability and based on the computational model by Durstewitz and Seamans (2008), more flexible persons should also be more distractible. While the univariate analysis of activation strength revealed no indication of greater reliance on brain systems critical for inhibitory processes during distractor inhibition in persons with higher spontaneous switching rates, functional connectivity analyses suggest that these persons do indeed couple the IFJ more strongly to brain systems known to be involved in attentional reorienting (TPJ; cf. Corbetta \& Shulman, 2002) and in supporting stimulus-oriented attending (anterior medial frontal cortex; cf. Burgess, Dumontheil, \& Gilbert, 2007). Thus, the spontaneous switching rate indeed modulates neural systems involved in both cognitive flexibility and cognitive stability. We interpret this result as a first piece of evidence in support of the dimensional model of cognitive flexibility and stability.
Importantly, we identified one brain area, that is, in the left superior frontal cortex, whose coupling to the IFJ was antagonistically modulated by the spontaneous switching rate depending on the currently required task dimension, that is, flexible task switching versus stable distractor inhibition. Most specifically, the coupling between this superior frontal region and the left IFJ was negatively correlated with the spontaneous switching rate during task switching and positively correlated with the spontaneous switching rate during distractor inhibition. Although the left SFG was not reliably activated nor deactivated in any of our task conditions, it is nevertheless very likely to play an important role for executive control functions: A lesion study investigating the left SFG in the context of an $n$-back task pointed out its importance for spatial working memory and also showed a general complexity effect, that is, increased relevance of the SFG with higher cognitive demands (du Boisgueheneuc et al., 2006). In the light of this finding, our results might point toward a general efficiency effect, that is, that with higher spontaneous switching rates less connectivity within a prefrontal executive control network is necessary for successful task switching, whereas higher connectivity is necessary to compensate for higher distractibility during distractor inhibition. Furthermore, a recent study from our lab showed the relevance of IFJSFG connectivity for switching of abstract task rules in contrast to switching of response hand, suggesting that IFJ-SFG coupling might reflect spatial rehearsal processes when switching the task rule (Stelzel, Basten, \& Fiebach, 2011; see also Curtis, Cole, Rao, \& D'Esposito, 2005).

There is, however, also evidence that the SFG is part of a task-negative network (or "default mode network") that is typically deactivated during task performance (Shirer, Ryali, Rykhlevskaia, Menon, \& Greicius, 2012; Harrison et al., 2011; Laird et al., 2011; Raichle et al., 2001). Negative correlations between neural activity in the task-negative and the task-positive network (i.e., the network that is generally activated during cognitive tasks) have been observed during rest as well as during task performance (Kelly, Uddin, Biswal, Castellanos, \& Milham, 2008; Fox et al., 2005; Greicius, Krasnow, Reiss, \& Menon, 2003). A study by Kelley and colleagues (2008) suggests that task performance, in the sense of low intraindividual variability, does benefit from a negative correlation between activity in the task-negative and task-positive networks during engagement in a task. However, methodological issues concerning the analysis of strength and direction of such correlations are currently a matter of debate (Hampson, Driesen, Roth, Gore, \& Constable, 2010; Murphy, Birn, Handwerker, Jones, \& Bandettini, 2009). A more recent study by Gordon, Stollstorff, Devaney, Bean, and Vaidya (2011) characterized connectivity only in relative terms: these authors found a dopaminergic effect on connectivity between the taskpositive and task-negative networks. Individuals with the 10/10 genotype of the DAT1 polymorphism—presumably characterized by higher striatal dopamine levels—-featured higher connectivity between specific regions of these two 
networks while performing a working memory task. These individuals also reported higher levels of impulsivity and inattention, even though there were no differences in task performance. The current results might represent further evidence for the relevance of coupling between the task-positive and the task-negative network for task performance: During distractor inhibition, more distractible persons show a presumably detrimental higher connectivity between a region of the task-positive network (the IFJ) and a region of the task negative network (the SFG). On the other hand, these persons might benefit from being more flexible during task switching — reflected in less connectivity between the two regions of the task-positive and tasknegative networks in the task switching condition of our task. Even though the SFG site identified here shows good overlap with task-negative networks reported in the literature, this interpretation is weakened by the fact that the SFG in our study did not show negative BOLD signals. This, however, may result from the specific paradigm that has as baseline an ongoing task and no prolonged periods of rest.

The observed IFJ-SFG coupling is the most direct neural reflection of the hypothesized unidimensional construct of cognitive stability versus flexibility and its operationalization by the spontaneous switching rate: While persons with a tendency toward spontaneous switching require less IFJ-SFG coupling to initiate a change of the attractor state during a switch trial, they need stronger IFJ-SFG coupling to prevent a spontaneous change of state during a distractor trial. Thus, by inference, these changes of functional coupling depending on task demands and the individual spontaneous switching rate can be regarded as a critical neural correlate of the individual disposition toward more flexible versus more stable cognitive control of goal-directed behavior.

\section{Acknowledgments}

This research was funded through a grant from the German Ministry of Education and Research (BMBF; 01GQ1003A). C. J. F. is also supported by the LOEWE initiative by the State of Hessen and by the Emmy Noether Program of the German Research Foundation (DFG FI 848/3-1).

Reprint requests should be sent to Diana J. N. Armbruster, Department of Psychology, Goethe University Frankfurt am Main, Mertonstr. 17, 60325 Frankfurt am Main, Germany, or via e-mail: armbruster@psych.uni-frankfurt.de.

\section{REFERENCES}

Aron, A. R., Monsell, S., Sahakian, B. J., \& Robbins, T. W. (2004). A componential analysis of task-switching deficits associated with lesions of left and right frontal cortex. Brain, 127, 1561-1573.

Aron, A. R., Robbins, T. W., \& Poldrack, R. A. (2004). Inhibition and the right inferior frontal cortex. Trends in Cognitive Sciences, 8, 170-177.

Banich, M. T. (2009). Executive function: The search for an integrated account. Current Directions in Psvchological Science, 18, 89-94.
Barber, A. D., \& Carter, C. S. (2005). Cognitive control involved in overcoming prepotent response tendencies and switching between tasks. Cerebral Cortex, 15, 899-912.

Bilder, R. M., Volavka, J., Lachman, H. M., \& Grace, A. A. (2004). The catechol-O-methyltransferase polymorphism: Relations to the tonic-phasic dopamine hypothesis and neuropsychiatric phenotypes. Neuropsychopharmacology 29, 1943-1961.

Brass, M., Derrfuss, J., Forstmann, B., \& von Cramon, D. Y. (2005). The role of the inferior frontal junction area in cognitive control. Trends in Cognitive Sciences, 9, 314-316.

Brunel, N., \& Wang, X. J. (2001). Effects of neuromodulation in a cortical network model of object working memory dominated by recurrent inhibition. Journal of Computational Neuroscience, 11, 63-85.

Burgess, P. W., Dumontheil, I., \& Gilbert, S. J. (2007). The gateway hypothesis of rostral prefrontal cortex (area 10) function. Trends in Cognitive Sciences, 11, 290-298.

Champod, A. S., \& Petrides, M. (2007). Dissociable roles of the posterior parietal and the prefrontal cortex in manipulation and monitoring processes. Proceedings of the National Academv of Sciences. U.S.A., 104, 14837-14842.

Collette, F., Salmon, E., Van der Linden, M., Chicherio, C., Belleville, S., Degueldre, C., et al. (1999). Regional brain activity during tasks devoted to the central executive of working memory. Cognitive Brain Research, 7, 411-417.

Collette, F., \& Van der Linden, M. (2002). Brain imaging of the central executive component of working memory. Neuroscience and Biobehavioral Reviews, 26, 105-125.

Compte, A., Brunel, N., Goldman-Rakic, P. S., \& Wang, X. J. (2000). Synaptic mechanisms and network dynamics underlying spatial working memory in a cortical network model. Cerebral Cortex, 10, 910-923.

Cools, R., Clark, L., Owen, A. M., \& Robbins, T. W. (2002). Defining the neural mechanisms of probabilistic reversal learning using event-related functional magnetic resonance imaging. Journal of Neuroscience, 22, 4563-4567.

Corbetta, M., \& Shulman, G. L. (2002). Control of goal-directed and stimulus-driven attention in the brain. Nature Reviews Neuroscience, 3, 201-215.

Cousineau, D. (2005). Confidence intervals in within-subject designs: A simpler solution to Loftus and Masson's method. Tutorial in Quantitative Methods for Psychology, 1, 42-45.

Curtis, C. E., Cole, M. W., Rao, V. Y., \& D'Esposito, M. (2005). Canceling planned action: An fMRI study of countermanding saccades. Cerebral Cortex, 15, 1281-1289.

Curtis, C. E., \& D’Esposito, M. (2003). Persistent activity in the prefrontal cortex during working memory. Trends in Cognitive Sciences, 7, 415-423.

Derrfuss, J., Brass, M., Neumann, J., \& von Cramon, D. Y. (2005). Involvement of the inferior frontal junction in cognitive control: Meta-analyses of switching and Stroop studies. Human Brain Mapping, 25, 22-34.

Derrfuss, J., Brass, M., \& von Cramon, D. Y. (2004). Cognitive control in the posterior frontolateral cortex: Evidence from common activations in task coordination, interference control, and working memory. Neuroimage, 23, 604-612.

Derrfuss, J., Brass, M., von Cramon, D. Y., Lohmann, G., \& Amunts, K. (2009). Neural activations at the junction of the inferior frontal sulcus and the inferior precentral sulcus: Interindividual variability, reliability, and association with sulcal morphology. Human Brain Matping 30, 299-311.

D'Esposito, M., Detre, J. A., Alsop, D. C., Shin, R. K., Atlas, S., \& Grossman, M. (1995). The neural basis of the central executive system of working memory. Nature, 378, 279-281.

Dodds, C. M., Morein-Zamir, S., \& Robbins, T. W. (2011). Dissociating inhibition, attention, and response control 
in the frontoparietal network using functional magnetic resonance imaging. Cerebral Cortex, 21, 1155-1165.

Dove, A., Pollmann, S., Schubert, T., Wiggins, C. J., \& von Cramon, D. Y. (2000). Prefrontal cortex activation in task switching: An event-related fMRI study. Brain Research. Cognitive Brain Research, 9, 103-109.

Dreher, J. C., Koechlin, E., Ali, S. O., \& Grafman, J. (2002). The roles of timing and task order during task switching. Neuroimage, 17, 95-109.

Dreisbach, G., \& Goschke, T. (2004). How positive affect modulates cognitive control: Reduced perseveration at the cost of increased distractibility. Lournal of Experimental Psychologv: Learning. Memory and Cognition, 30, 343-353.

Dreisbach, G., Muller, J., Goschke, T., Strobel, A., Schulze, K., Lesch, K. P., et al. (2005). Dopamine and cognitive control: The influence of spontaneous eyeblink rate and dopamine gene polymorphisms on perseveration and distractibility. Behavioral Neuroscience, 119, 483-490.

du Boisgueheneuc, F., Levy, R., Volle, E., Seassau, M., Duffau, H., Kinkingnehun, S., et al. (2006). Functions of the left superior frontal gyrus in humans: A lesion study. Brain, 129, 3315-3328.

Duncan, J., \& Owen, A. M. (2000). Common regions of the human frontal lobe recruited by diverse cognitive demands. Trends in Neurosciences, 23, 475-483.

Durstewitz, D., Kelc, M., \& Gunturkun, O. (1999). A neurocomputational theory of the dopaminergic modulation of working memory functions. Journal of Neuroscience, 19, 2807-2822.

Durstewitz, D., \& Seamans, J. K. (2002). The computational role of dopamine D1 receptors in working memory. Neural Networks, 15, 561-572.

Durstewitz, D., \& Seamans, J. K. (2008). The dual-state theory of prefrontal cortex dopamine function with relevance to catechol-o-methyltransferase genotypes and schizophrenia. Biological Psvchiatrv, 64, 739-749.

Durstewitz, D., Seamans, J. K., \& Sejnowski, T. J. (2000). Neurocomputational models of working memory. Nature Neuroscience, 3(Suppl.), 1184-1191.

Faisal, A. A., Selen, L. P., \& Wolpert, D. M. (2008). Noise in the nervous system. Nature Reviews Neuroscience, 9, 292-303.

Forman, S. D., Cohen, J. D., Fitzgerald, M., Eddy, W. F., Mintun, M. A., \& Noll, D. C. (1995). Improved assessment of significant activation in functional magnetic resonance imaging (fMRI): Use of a cluster-size threshold. Magnetic Resonance in Medicine, 33, 636-647.

Forstmann, B. U., Jahfari, S., Scholte, H. S., Wolfensteller, U., van den Wildenberg, W. P., \& Ridderinkhof, K. R. (2008). Function and structure of the right inferior frontal cortex predict individual differences in response inhibition: A model-based approach. Journal of Neuroscience, 28, 9790-9796.

Fox, M. D., Snyder, A. Z., Vincent, J. L., Corbetta, M., Van Essen, D. C., \& Raichle, M. E. (2005). The human brain is intrinsically organized into dynamic, anticorrelated functional networks. Proceedings of the National Academv of Sciences. U.S.A., 102, 9673-9678.

Friston, K. J., Buechel, C., Fink, G. R., Morris, J., Rolls, E., \& Dolan, R. J. (1997). Psychophysiological and modulatory interactions in neuroimaging. Neuroimage, 6, 218-229.

Friston, K. J., Fletcher, P., Josephs, O., Holmes, A., Rugg, M. D., \& Turner, R. (1998). Event-related fMRI: Characterizing differential responses. Neuroimage, 7, 30-40.

Gordon, E. M., Stollstorff, M., Devaney, J. M., Bean, S., \& Vaidya, C. J. (2011). Effect of dopamine transporter genotype on intrinsic functional connectivity depends on cognitive state. Cerebral Cortex, 22, 2182-2196.
Greicius, M. D., Krasnow, B., Reiss, A. L., \& Menon, V. (2003). Functional connectivity in the resting brain: A network analysis of the default mode hypothesis. Proceedings of the National Academv of Sciences. U.S.A., 100, 253-258.

Hampson, M., Driesen, N., Roth, J. K., Gore, J. C., \& Constable, R. T. (2010). Functional connectivity between task-positive and task-negative brain areas and its relation to working memory performance. Magnetic Resonance Imaging, 28, 1051-1057.

Harrison, B. J., Pujol, J., Contreras-Rodriguez, O., Soriano-Mas, C., Lopez-Sola, M., Deus, J., et al. (2011). Task-induced deactivation from rest extends beyond the default mode brain network. PLoS One, 6, e22964.

Hedden, T., \& Gabrieli, J. D. (2010). Shared and selective neural correlates of inhibition, facilitation, and shifting processes during executive control. Neuroimage, 51, 421-431.

Hedden, T., \& Yoon, C. (2006). Individual differences in executive processing predict susceptibility to interference in verbal working memory. Neuropsychology, 20, 511-528.

Jersild, A. (1927). Mental set and shift. Archives of Psychology, 14, 81.

Jha, A. P., Fabian, S. A., \& Aguirre, G. K. (2004). The role of prefrontal cortex in resolving distractor interference. Cognitive. Affective, and Behavioral Neuroscience, 4, 517-527.

Kellendonk, C., Simpson, E. H., Polan, H. J., Malleret, G., Vronskaya, S., Winiger, V., et al. (2006). Transient and selective overexpression of dopamine D2 receptors in the striatum causes persistent abnormalities in prefrontal cortex functioning. Neuron, 49, 603-615.

Kelly, A. M., Uddin, L. Q., Biswal, B. B., Castellanos, F. X., \& Milham, M. P. (2008). Competition between functional brain networks mediates behavioral variability. Neuroimage. 39, 527-537.

Konishi, S., Nakajima, K., Uchida, I., Kikyo, H., Kameyama, M., \& Miyashita, Y. (1999). Common inhibitory mechanism in human inferior prefrontal cortex revealed by event-related functional MRI. Brain, 122, 981-991.

Laakso, A., Pohjalainen, T., Bergman, J., Kajander, J., Haaparanta, M., Solin, O., et al. (2005). The A1 allele of the human D2 dopamine receptor gene is associated with increased activity of striatal L-amino acid decarboxylase in healthy subjects. Pharmacogenetics and Genomics, 15, 387-391.

Laird, A. R., Fox, P. M., Eickhoff, S. B., Turner, J. A., Ray, K. L., McKay, D. R., et al. (2011). Behavioral interpretations of intrinsic connectivity networks. Journal of Cognitive Neuroscience, 23, 4022-4037.

Loh, M., Rolls, E. T., \& Deco, G. (2007). A dynamical systems hypothesis of schizophrenia. PLoS Computational Biology, 3, e228.

Meiran, N., Chorev, Z., \& Sapir, A. (2000). Component processes in task switching. Cognitive Psvchology, 41, 211-253.

Miller, E. K., \& Cohen, J. D. (2001). An integrative theory of prefrontal cortex function. Annual Review of Neuroscience, 24, 167-202.

Miyake, A., Friedman, N. P., Emerson, M. J., Witzki, A. H., Howerter, A., \& Wager, T. D. (2000). The unity and diversity of executive functions and their contributions to complex "frontal lobe" tasks: A latent variable analysis. Cognitive Psychology, 41, 49-100.

Monsell, S. (2003). Task switching. Trends in Cognitive Sciences, 7, 134-140.

Muller, J., Dreisbach, G., Brocke, B., Lesch, K. P., Strobel, A., \& Goschke, T. (2007). Dopamine and cognitive control: The influence of spontaneous eyeblink rate, DRD4 exon III 
polymorphism and gender on flexibility in set-shifting. Brain Research, 1131, 155-162.

Murphy, K., Birn, R. M., Handwerker, D. A., Jones, T. B., \& Bandettini, P. A. (2009). The impact of global signal regression on resting state correlations: Are anti-correlated networks introduced? Neuroimage, 44, 893-905.

Nichols, T., Brett, M., Andersson, J., Wager, T., \& Poline, J. B. (2005). Valid conjunction inference with the minimum statistic. Neuroimage, 25, 653-660.

Pollmann, S., Dove, A., Yves von Cramon, D., \& Wiggins, C. J. (2000). Event-related fMRI: Comparison of conditions with varying BOLD overlap. Human Brain Mapping, 9, 26-37.

Raichle, M. E., MacLeod, A. M., Snyder, A. Z., Powers, W. J., Gusnard, D. A., \& Shulman, G. L. (2001). A default mode of brain function. Proceedings of the National Academv of Sciences. U.S.A., 98, 676-682.

Robbins, T. W. (2007). Shifting and stopping: Fronto-striatal substrates, neurochemical modulation and clinical implications. Philosophical Transactions of the Royal Society of London, Series B, Biological Sciences, 362, 917-932.

Rolls, E. T., \& Deco, G. (2010). The noisy brain: Stochastic dynamics as a principle of brain function. Oxford: Oxford University Press.

Rolls, E. T., Loh, M., Deco, G., \& Winterer, G. (2008). Computational models of schizophrenia and dopamine modulation in the prefrontal cortex. Nature Reviews Neuroscience, 9, 696-709.

Rosa, E. C., Dickinson, D., Apud, J., Weinberger, D. R., \& Elvevag, B. (2010). COMT Val158Met polymorphism, cognitive stability and cognitive flexibility: An experimental examination. Behavioral and Brain Functions, 6, 53.

Sakai, K., Rowe, J. B., \& Passingham, R. E. (2002). Active maintenance in prefrontal area 46 creates distractor-resistant memory. Nature Neuroscience, 5, 479-484.

Shirer, W. R., Ryali, S., Rykhlevskaia, E., Menon, V., \& Greicius, M. D. (2012). Decoding subject-driven cognitive states with wholebrain connectivity patterns. Cerebral Cortex, 22, 158-165.

Stelzel, C., Basten, U., \& Fiebach, C. J. (2011). Functional connectivity separates switching operations in the posterior lateral frontal cortex. Iournal of Cognitive Neuroscience, 23, 3529-3539.

Stelzel, C., Basten, U., Montag, C., Reuter, M., \& Fiebach, C. J. (2009). Effects of dopamine-related gene-gene interactions on working memory component processes. European Iournal of Neuroscience, 29, 1056-1063.
Stelzel, C., Basten, U., Montag, C., Reuter, M., \& Fiebach, C. J. (2010). Frontostriatal involvement in task switching depends on genetic differences in $\mathrm{d} 2$ receptor density. Lournal of Neuroscience, 30, 14205-14212.

Strogatz, S. H. (2001). Nonlinear dynamics and chaos: With applications to physics, biology, chemistry, and engineering. Boulder, CO: Westview Press.

Swick, D., Ashley, V., \& Turken, U. (2011). Are the neural correlates of stopping and not going identical? Quantitative meta-analysis of two response inhibition tasks. Neuroimage. 56, 1655-1665.

Thurley, K., Senn, W., \& Luscher, H. R. (2008). Dopamine increases the gain of the input-output response of rat prefrontal pyramidal neurons. Journal of Neurophysiology, 99, 2985-2997.

Toepper, M., Gebhardt, H., Beblo, T., Thomas, C., Driessen, M., Bischoff, M., et al. (2010). Functional correlates of distractor suppression during spatial working memory encoding. Neuroscience, 165, 1244-1253.

van Holstein, M., Aarts, E., van der Schaaf, M. E., Geurts, D. E., Verkes, R. J., Franke, B., et al. (2011). Human cognitive flexibility depends on dopamine D2 receptor signaling. Psychopharmacology, 218, 567-578.

Wager, T. D., Jonides, J., \& Reading, S. (2004). Neuroimaging studies of shifting attention: A meta-analysis. Neuroimage, 22, 1679-1693.

Wager, T. D., Jonides, J., Smith, E. E., \& Nichols, T. E. (2005). Toward a taxonomy of attention shifting: Individual differences in fMRI during multiple shift types. Cognitive. Affective and Behavioral Neuroscience, 5, 127-143.

Wager, T. D., \& Smith, E. E. (2003). Neuroimaging studies of working memory: A meta-analysis. Cognitive. Affective. and Behavioral Neuroscience, 3, 255-274.

Wang, X. J. (1999). Synaptic basis of cortical persistent activity: The importance of NMDA receptors to working memory. Journal of Neuroscience, 19, 9587-9603.

Ward, B. D. (2000). Simultaneous inference for fMRI data. Retrieved from afni.nimh.nih.gov/pub/dist/doc/manual/ AlphaSim.pdf.

Winterer, G., Musso, F., Beckmann, C., Mattay, V., Egan, M. F., Jones, D. W., et al. (2006). Instability of prefrontal signal processing in schizophrenia. American Iournal of Psvchiatrv, 163, 1960-1968.

Winterer, G., \& Weinberger, D. R. (2004). Genes, dopamine and cortical signal-to-noise ratio in schizophrenia. Trends in Neurosciences, 27, 683-690. 


\section{This article has been cited by:}

1. Paul S. Muhle-Karbe, Wouter De Baene, Marcel Brass. 2014. Do tasks matter in task switching? Dissociating domain-general from context-specific brain activity. NeuroImage 99, 332-341. [CrossRef]

2. Thomas Goschke, Annette Bolte. 2014. Emotional modulation of control dilemmas: The role of positive affect, reward, and dopamine in cognitive stability and flexibility. Neuropsychologia 62, 403-423. [CrossRef]

3. S. Helene Richter, Anne S. Vogel, Kai Ueltzh Ãđffer, Chiara Muzzillo, Miriam A. Vogt, Katja Lankisch, Diana J. N. ArmbrusterGenÃ§, Marco A. Riva, Christian J. Fiebach, Peter Gass, Barbara Vollmayr. 2014. Touchscreen-paradigm for mice reveals crossspecies evidence for an antagonistic relationship of cognitive flexibility and stability. Frontiers in Bebavioral Neuroscience 8. . [CrossRef]

4. Thomas Goschke. 2014. Dysfunctions of decision-making and cognitive control as transdiagnostic mechanisms of mental disorders: advances, gaps, and needs in current research. International Journal of Methods in Psychiatric Research 23:10.1002/ mpr.v23.S1, 41-57. [CrossRef] 